\title{
Evaluation of Inoculation Methods to Reproduce Sunflower Premature Ripening Caused by Phoma macdonaldii
}

\author{
C. Seassau and P. Debaeke, INRA, UMR AGIR, BP 52627, F-31326 Castanet-Tolosan cedex, France; E. Mestries, \\ CETIOM, ENSAT, BP 32607, F-31326 Castanet-Tolosan cedex, France; and G. Dechamp-Guillaume, ENSAT, \\ INPT, UMR AGIR, BP 32607, F-31326 Castanet-Tolosan cedex, France
}

\begin{abstract}
Seassau, C., Debaeke, P., Mestries, E., and Dechamp-Guillaume, G. 2010. Evaluation of inoculation methods to reproduce sunflower premature ripening caused by Phoma macdonaldii. Plant Dis. 94:1398-1404.

Three inoculation methods were evaluated for effectiveness to cause sunflower premature ripening (PR). Evaluations were conducted on a sunflower (Helianthus annuus) cultivar susceptible to PR in replicated, multilocation experiments under greenhouse conditions. Plants were inoculated with Phoma macdonaldii, either with mycelium, conidia, or infected residues at the stem base or with buried residues. Disease severity (DS) was measured by percent girdling necrosis at the stem base and percent final PR; the infection spread was assessed using the area under the disease progress curve (AUDPC). Inoculation with mycelia or $1 \times 10^{6} \mathrm{spores} / \mathrm{ml}$ caused significantly more DS and PR than lower spore concentrations or infected residues $(P<0.05)$. Amending soil with residues induced root necrosis but no PR. P. macdonaldii was mainly isolated at the stem base and above but rarely on root systems. Microscopic evaluations showed that hyphae colonized mainly the cortex and vascular stem tissues. The overall results demonstrated a clear role of aerial infection in PR compared with soilborne inoculum, and that inoculation at the stem base with a spore suspension could be used for screening genotypes for resistance to PR.
\end{abstract}

Sunflower premature ripening (PR) is regarded as one of the most widespread and detrimental diseases in sunflower (Helianthus annuus L.) production in France, where moderate to severe outbreaks have occurred over the past decade. The incidence and severity of the disease increased dramatically in the early 1990s, and the entire French sunflower cropping area is now affected $(18,20)$. Epidemics have caused extensive damage with direct losses in grain yield in France of up to 1.3 t/ha (23). Phoma macdonaldii Boerema (3) (teleomorph Leptosphaeria lindquistii Frezzi) (8) has been reported as the causal agent of sunflower PR (15). The pathogen has been observed in western and eastern Europe (13,18), southern Asia (31), Argentina (30), Australia (16), and the United States (7). Sackston (28) first described a wilt and stalk rot of unknown etiology as "premature ripening". Early research showed that a girdling canker at the base of the stem caused by P. macdonaldii was

Corresponding author: G. Dechamp-Guillaume E-mail: dechamp@ensat.fr

Accepted for publication 2 August 2010.

doi:10.1094/PDIS-03-10-0180

(C) 2010 The American Phytopathological Society the primary cause of premature ripening. From mid- to late summer, leaves become wilted and necrotic, and the stalk turns dark brown to black leading to plant death a few weeks before physiological maturity (7).

Sunflower residues have long been considered the major source of primary inoculum in $P$. macdonaldii epidemics. Short rotations (mainly sunflower-wheat-sunflower) and reduced tillage of wheat crops could have increased the inoculum by leaving infected residues on the soil surface. Primary inoculum originates from overwintering fungal structures (perithecia, pycnidia, and mycelia) in crop debris, which are spread to healthy plants by rain splash and windblown ascospores and conidia produced during wet conditions (10). Perithecia and pycnidia have been observed on sunflower stalk residues even several years after harvest (14), and buried residues brought back to the soil surface still retained viable inoculum (24). However, inoculations from infected buried residues were suggested to result in less infection than residues left on the soil surface (20). Although spores were reported to be pathogenic $(19,22)$, uncertainty remains about the relative importance of conidia, ascospores, and mycelium in causing sunflower PR.

Donald et al. (7) first observed that root systems of girdled plants were poorly de- veloped and necrotic, and $P$. macdonaldii was isolated from the basal stalk tissue of PR plants. In contrast, Pérès and Poisson (22) found that roots of girdled plants were well developed and $P$. macdonaldii was rarely isolated from roots. There is controversy about whether PR originates as the result of root infection (soilborne) or from stem base infection (aerial). It is not known whether PR is due to direct root infection, to basal stem infection progressing toward the roots, or to basal stem girdling inhibiting nutrient transport into the roots. Moreover, recent investigations indicated factors such as additional nitrogen (6) and limited water supply after anthesis can significantly influence the incidence of P. macdonaldii attacks and PR (29). PR was also greatest when plants were inoculated at star bud stage, and decreased considerably when plants were inoculated at earlier or later growth stages (21).

The objectives of this study were (i) to assess the efficiency of artificial inoculation methods (from infected residues, mycelium, or conidia) in reproducing PR on a cultivar susceptible to $P$. macdonaldii in a controlled environment, and (ii) to determine whether $\mathrm{PR}$ is from root infection and spread of the pathogen into the stem, or directly from stem base infection leading to girdling, plant wilting, and death.

\section{MATERIALS AND METHODS}

Experimental design and crop management systems. Five greenhouse experiments were conducted during a 5month period: at INRA, Auzeville (HauteGaronne, France) in 2007 (Auz-07), in 2008 (Auz-08), and in 2009 (Auz-09); at CETIOM En-Crambade (Haute-Garonne, France) in 2007 (EC-07); and at Grignon (Yvelines, France) in 2008 (Gri-08). Seeds of cv. Heliasol RM, a commercial sunflower cultivar, were obtained from Semences de France (KWS AG). This cultivar was susceptible to $P$. macdonaldii and PR based on previous field trials (29). Seeds were surface disinfected in $9 \%$ sodium hypochlorite for $5 \mathrm{~min}$ and rinsed four times in sterile, distilled water. Four seeds were sown in 15-liter pots filled with a mixture of $50 \%$ clay to silt-clay soil (from a field uncultivated for more than 30 
years), $40 \%$ potting compost, and $10 \%$ sand at Auzeville and Grignon, and in 12liter pots filled with $60 \%$ potting compost and $40 \%$ sand at En-Crambade.

In the greenhouses at Auzeville and Grignon, photoperiod, temperature, and air humidity were controlled; while at EnCrambade, the experiment was carried out in a hoop tunnel where those factors were not controlled. A 14-h photoperiod was applied from plant emergence to the star bud stage with $400 \mathrm{~W}$ High Pressure Sodium vapor lamp (SON-T AGRO, Philips) at Auzeville and at Grignon (Blanc Europe MBS, Claude). Supplemental lighting was turned off when global radiation was above $250 \mathrm{~W} / \mathrm{m}^{2}$. Temperature in the greenhouses was maintained at $17^{\circ} \mathrm{C}$ at night and $25 \pm$ $5^{\circ} \mathrm{C}$ during the day. Plants were fertilized with three applications of NPK solution (50 kg N, $20 \mathrm{~kg} \mathrm{P}, 20 \mathrm{~kg} \mathrm{~K} / \mathrm{ha}$ ) until the star bud stage. Plants were irrigated daily by a $2-l i t e r / h$ emitter to maintain adequate soil moisture until flowering, and then the water supply was reduced. The fraction of transpirable soil water was kept between 30 and $50 \%$ by weighing plants every 2 days and adding water as needed. Aphids were treated with tau-fluvalinate (Mavrik Flo, Makhteshim Agan France) and pyrimicarb (Pirimor G, Syngenta Agro SAS) at rates of $72 \mathrm{~g}$ a.i./ha and $500 \mathrm{~g}$ a.i./ha, respectively. Citrus whiteflies (Aleyrodidae) were treated by pymetrozin (Chess Pro, Syngenta Agro SAS) at a rate of $40 \mathrm{~g}$ a.i./ha at Grignon. Powdery mildew (Erysiphe cichoracearum) was treated by wettable sulfur (Phytosoufre, Bayer France SAS) at $10 \mathrm{~g} /$ liter.

Fungal isolates and inoculum. A single conidium culture of $P$. macdonaldii (MPH2 strain), selected for its severe aggressiveness, was used in this study. The strain was isolated from infected sunflower stem residue collected near Auzeville in 2006 and stored at $-80^{\circ} \mathrm{C}$ in glycerol.

For the Auz-07 and Gri-08 experiments, sunflower residues infected with $P$. macdonaldii were collected in January 2007 from a field close to the experimental site. In the EC-07 experiment, the residue was collected in mid-March 2007 on plots inoculated with $P$. macdonaldii during the 2006 season. Following collection, infected residues were placed outside on short grass to allow the pathogen to complete the life cycle. Prior to use as inoculum, stalk residues showing $P$. macdonaldii pycnidia and visually devoid of other fungi were slit open and pith was removed. Mycelium was derived from the MPH2 culture. The fungus was grown for 10 days in the dark at $25 \pm 1{ }^{\circ} \mathrm{C}$ on potato dextrose agar (PDA) (Difco) amended with 150 $\mathrm{mg} /$ liter of streptomycin ( $\mathrm{pH} 6$ ).

Spores were produced from the MPH2 culture. The fungus was grown on PDA at $25 \pm 1{ }^{\circ} \mathrm{C}$ in alternating 12 -h light and dark cycles for 15 days to induce pycnidia. Spores were harvested by placing $1 \mathrm{~cm}^{2}$ of
PDA with pycnidia in a petri dish containing sterile, distilled water. The concentration of conidia in suspension was determined using an hemacytometer and adjusted with sterile, distilled water to $1 \times$ $10^{6}$ spores $/ \mathrm{ml}$.

Infected residues and mycelium inoculum experiment. Three experiments were carried out to evaluate the relative importance of infected residues and mycelium on PR incidence. Inoculation methods were: (i) a 6-mm-diameter disk of PDA with $P$. macdonaldii mycelium was placed at the stem base, and drying of the disk was prevented by applying moist cotton over the mycelium and covering with aluminum foil; (ii) 10 fragments of stalk residues, 10 to $15 \mathrm{~cm}$ long, were randomly buried per pot; and (iii) 10 residue fragments were placed on the soil surface per pot. In Gri-08, the three methods were tested on cv. Heliasol RM. In Auz-07 and EC-07, inoculation methods (i) and (ii) were tested, except that in method (ii), some fragments of residues remained at the soil surface. In Gri-08, residues remaining at the soil surface were sprayed with water twice a week to maintain the moisture required for spore production. The burial of residues in method (ii) was prior to planting. Stem base inoculation with a mycelium disk (i) as well as positioning of residues on the soil surface (iii) were completed at star bud stage. The mycelium disk was left for 7 days, and residues remained throughout the experiment.

The experimental design was a randomized complete block with three blocks for Auz-07 and Gri-08 and four blocks for EC-07. Eight plants (Auz-07, Gri-08) and 20 plants (EC-07) per block were evaluated for each inoculation method and for control treatment without residues.

Conidia dose-response experiment. To determine the amount and type of inoculum required for maximizing disease incidence and premature ripening, four concentrations $\left(1 \times 10^{3}, 1 \times 10^{4}, 1 \times 10^{5}\right.$, and $1 \times 10^{6}$ spores $/ \mathrm{ml}$ ) of conidia in suspension were evaluated in comparison with a mycelium inoculation in Auz-08, and $1 \times 10^{6}$ spores/ml was compared with a mycelium inoculation in Auz-09. Spore concentrations were prepared just prior to inoculations. The experiment was a randomized complete block design with four blocks. Eight plants per block were evaluated for each inoculation method and disease-free treatment (control). The methods used were: (i) a 6-mm-diameter disk of PDA with MPH2 mycelium placed at the stem base with moist cotton covered with aluminum foil, and (ii) $70 \mu \mathrm{l}$ of a conidia suspension pipetted onto a 6-mm-diameter disk of sterilized cellulose, applied at the stem base and held with Parafilm M. Control plants received a disk with $70 \mu$ of sterile distilled water placed on the stem base and held with Parafilm M. Plants were inoculated at star bud stage with a mycelium or conidia suspension. Mycelia or disks with spores were maintained on the stem base for 7 days.

Isolation. Isolations of $P$. macdonaldii were performed on a subsample of 35 and 23 sunflower plants inoculated with mycelium or buried residues, showing Phoma symptoms or not, in Auz-07 and EC-07, respectively. Roots were rinsed to remove soil prior to isolations. Small tissue samples were removed from the stem base, the stem at 4 to $5 \mathrm{~cm}$ above stem base necrosis, and roots. Samples from Auz-07 were collected from the epidermis, cortex, and pith of the stem base and further up on the stem. Root samples included root hairs, rhizoderm, and cortex. Samples collected at EC-07 included the epidermis and pith of the stem base, and root hairs. Samples were surface disinfected in $1 \%$ sodium hypochlorite for $1 \mathrm{~min}$, followed by three 1-min rinses with sterile distilled water, and blotted dry before isolations. Then tissues were placed onto PDA amended with $150 \mathrm{mg} /$ liter of streptomycin $(\mathrm{pH} \mathrm{6})$ and incubated for 10 days at $25 \pm 1{ }^{\circ} \mathrm{C}$ under alternating light-dark periods to induce sporulation.

Sample preparation for microscopy. A sample of plants inoculated with $P$. macdonaldii mycelium from the Auzeville experiment was chosen. Because of tissue hardness, slices of stem, stem base, and roots were cut in 6 cross-sections. Then tissues were sectioned (130 to $140 \mu \mathrm{m}$ thick) on a vibratome (Series 1000, TPI, St. Louis, MO, USA) with a steel knife, and each slice was flooded with lactophenol cotton blue for $1 \mathrm{~min}$ and rinsed with distilled water. Image acquisition was performed at $\times 50$ to $\times 400$ with a CCD camera (color Coolview, Photonic Science, Robertsbridge, UK) mounted on an inverted microscope (DMIRBE, Leica, Reuil-Malmaison, France).

Disease assessment and data analyses. The development of necrotic areas at the stem base induced by $P$. macdonaldii inoculation was assessed regularly using a visual 0 to 3 scale: $0=$ healthy plant, 1 $=$ less than $3 / 4$ of the stem base circumference black, 2 = lesions girdling the stem base, 3 = all leaves wilted and plant completely dead. Disease severity (DS) was the percentage of plants with Phoma girdling necrosis at the stem base of the plant (scale 2), and premature ripening (PR) was the percentage of plants completely dry before physiological maturity with lesions girdling the stem base (scale 3 ). In experiments with infected residues and mycelium inoculum, the assessment was made weekly from inoculation up to 83 days postinoculation (DPI). In the conidia dose-response experiment, eight recordings were taken up to 78 DPI. DS and PR were assessed by the area under the disease progress curve (AUDPC). $\mathrm{AUDPC}_{\mathrm{DS}}$ and $\mathrm{AUDPC}_{\mathrm{PR}}$ were calculated 
according to the equation of Campbell and Madden (4):

$$
\text { AUDPC }=\sum_{i}^{n-1}\left(y_{i}+y_{i+1}\right) / 2 *\left(t_{i+1}-t_{i}\right)
$$

where $n$ is the number of evaluations, $y$ the DS or the percentage of PR, and $t$ the DPI of each evaluation. Besides AUDPC ${ }_{D S}$ and $\mathrm{AUDPC}_{\mathrm{PR}}$, the final percentage of PR sun- flowers was estimated at the end of each disease assessment experiment.

The independent variables $\mathrm{AUDPC}_{\mathrm{DS}}$, $\mathrm{AUDPC}_{\mathrm{PR}}$, and the final percentage of PR plants were analyzed by analysis of variance (ANOVA) via the general linear model procedure of Statgraphics Plus 5.1 statistical software (Rockville, MA, USA) with replicate as a random variable. For each ANOVA, homogeneity of variance by Levene's test (confidence level of 0.95) and the normality of the residuals by the Shapiro-Wilks test (confidence level of 0.95) were conducted. Prior to ANOVA, square-root normalizing transformations were carried out to $\mathrm{AUDPC}_{\mathrm{DS}}$ and
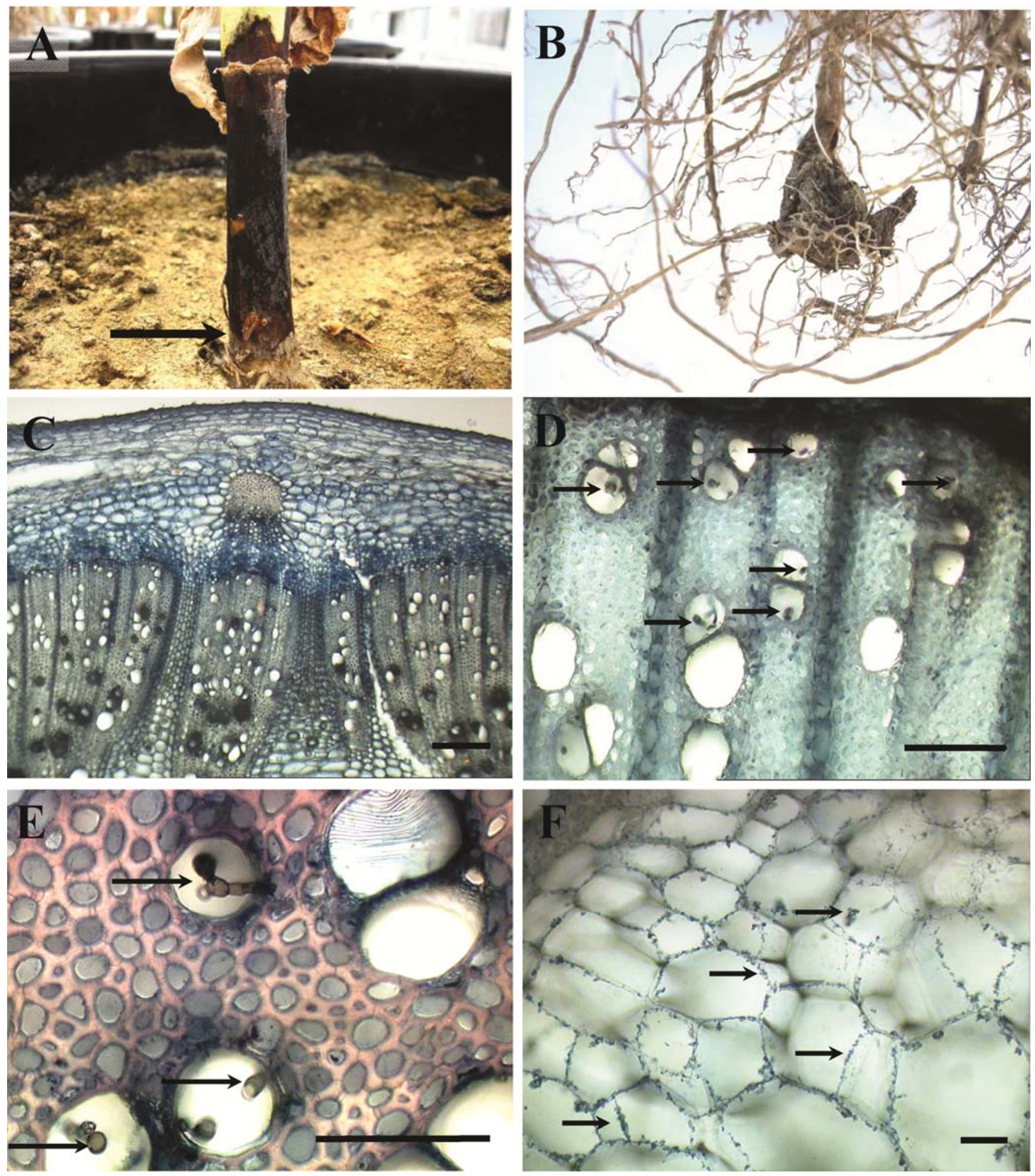

Fig. 1. A and B, Stem and root lesions on sunflower cv. Heliasol RM after artificial inoculation with Phoma macdonaldii. A, Phoma stem girdling necrosis after mycelium inoculation at stem base (arrow) with spreading of the fungus to the stem, and $\mathbf{B}$, black swollen necrotic areas on secondary roots of plants grown with P. macdonaldii-infested buried residue. C, Stem base cross-section of cv. Heliasol RM from noninoculated plants. Bar $=250 \mu \mathrm{m}$. D to F, Stem base cross-section of plants inoculated with P. macdonaldii mycelium. D, Secondary xylem tissues colonized with P. macdonaldii hyphae (arrow). Bar $=100 \mu \mathrm{m}$. E, Primary xylem tissues colonized with P. macdonaldii hyphae. Bar $=100 \mu \mathrm{m}$. F, Pith tissues extensively colonized with P. macdonaldii hyphae $(\operatorname{arrow})$. Bar $=100 \mu \mathrm{m}$. 
$\mathrm{AUDPC}_{\mathrm{PR}}$, and arcsine normalizing transformations were applied to the final percentage of DS and PR in infected residues and mycelium inoculum experiment (9). Where the $F$ ratio was significant $(P<$ 0.05 ), differences between treatment means were determined using protected least significant differences (LSD). Linear correlation (Pearson's coefficient of correlation) between DS and PR was determined to measure the degree of association between pairs of variables. In the experiment with conidia and mycelium inoculations, expected versus observed plant PR at 83 DPI was analyzed with a logistic model.

\section{RESULTS}

Infected residues and mycelium inoculation. Disease symptoms at the stem base became apparent 7 days after inoculation with mycelium disks, and all plants showed upwardly expanding necrotic lesions, extending as much as $15 \mathrm{~cm}$ on the stem (Fig. 1A). Plants grown with infected residues did not consistently exhibit symptoms on the stem base or roots. Inoculation with buried residues and a few fragments left on the soil surface in Auz-07 and EC07 induced 5.2 and $3.3 \%$ black lesions on the stem base, respectively. In the Gri-08 experiment, only $8.3 \%$ of plants grown with buried infected residues developed stem base necrosis, whereas inoculation with residues left on the soil surface resulted in $78.3 \%$ of plants with such symptoms. The secondary roots of some plants inoculated with buried residues showed swollen necrotic areas where the roots were in direct contact with the residues (Fig. 1B). The three experimental sites did not differ significantly in $\operatorname{AUDPC}_{\mathrm{DS}}(P=$ 0.9357), final DS $(P=0.8775)$, or $\operatorname{AUDPC}_{\mathrm{PR}}(P=0.0906)$ and final PR $(P=$ 0.4937). However, these variables were affected by the inoculation method. Inoculation with $P$. macdonaldii mycelium significantly $(P<0.0001)$ increased AUDPC ${ }_{D S}$ and DS compared to the other treatment (Table 1). Plants inoculated with residues on the soil surface in Gri-08 had significantly $(P<0.0001)$ higher AUDPC $\mathrm{DS}_{\mathrm{DS}}$ and DS than plants inoculated with buried residues in the other two sites. These differences between inoculation methods were observed also for $\mathrm{AUDPC}_{\mathrm{PR}}$ and the final PR. Inoculation with mycelium at the stem base induced significantly $(P<$ 0.0001) higher $\mathrm{AUDPC}_{\mathrm{PR}}$ and final $\mathrm{PR}$, compared to inoculation with infected residues on the soil surface (Gri-08) and buried residues in Auz-07. There was poor disease development on plants with buried residues in EC-07, and plants in the same treatment in Gri-08 remained symptomless like the controls. All plants with PR showed girdling stem base lesions, and Pearson's correlation coefficient for the percentage of plants with girdling stem base lesions and PR at 83 DPI was $r=$ $0.971(P<0.001)$ for Auz-07, $r=0.887(P$ $=0.0001)$ for EC-07, and $r=0.994(P<$ $0.0001)$ for Gri-08.

A $\chi^{2}$ analysis indicated that, for each location, either inoculation treatment significantly affected plant mortality $(P=0.0015$ for Auzeville and $P<0.0001$ for EnCrambade). Mycelium inoculation induced the highest plant mortality, while only some plants inoculated with buried residues showed girdling stem base lesions. $P$. macdonaldii was frequently isolated at the leading edge of the lesion and within the stem when plants had stem base lesions (Table 2). The presence of the pathogen was mainly observed on the epidermis and in the cortex of the stem base and above. In contrast, $P$. macdonaldii was seldom isolated from root hairs, rhizoderm, and root cortex. In plants inoculated with buried residues that did not show stem base lesions, $P$. macdonaldii was not isolated from pith. Noninoculated control plants remained symptomless and $P$. macdonaldii was not recovered.
Microscopic evaluations were performed on thin sections of stem (base and above) and root tissues after artificial inoculation with $P$. macdonaldii mycelium from Auz-07 site. At the leading edge of the infection, hyphae were abundant in the pith, primary and secondary xylem, cambium, phloem, and cortex of girdling lesions at the stem base and above (Fig. 1D to F). Microscopic observations on green tissues of the stem above the lesion showed that hyphae were mainly present in the primary xylem and the pith (Fig. 1E and F) and were not observed in the epidermis and within the cortex. Hyphae were not observed in root tissues.

Conidia and mycelium inoculation. Necrotic stem base lesions appeared as early as 7 days after inoculation with $P$. macdonaldii conidia suspensions and mycelium. Necrotic lesions could extend several centimeters above the initial spot of artificial inoculation, whatever the methods of inoculation. However, plants inoculated with low concentrations of spores (1 $\times 10^{3}$ and $1 \times 10^{4}$ spores $/ \mathrm{ml}$ ) had small lesions that spread slowly compared with plants inoculated with higher concentrations $\left(1 \times 10^{5}\right.$ and $1 \times 10^{6}$ spores $\left./ \mathrm{ml}\right)$ and mycelium. Disease severity was affected by inoculation method and spore concentration. From 43 days postinoculation (DPI), DS of plants with Phoma girdling increased significantly $(P=0.0012)$ when plants were inoculated with $1 \times 10^{5}$ spores $/ \mathrm{ml}, 1 \times 10^{6}$ spores $/ \mathrm{ml}$, and mycelium (Fig. 2). More than $92 \%$ of plants inoculated with mycelium or $1 \times 10^{6}$ spores/ml had girdling stem base lesions, and no significant differences were observed between the two methods. In contrast, plants inoculated with $1 \times 10^{3}$ spores/ml had the lowest DS with $14 \%$ at 78 DPI.

Premature ripened plants were observed from 55 DPI with inoculation $\geq 1 \times 10^{4}$ spores/ml and mycelium and from 66 DPI

Table 1. Evaluation of inoculation methods with Phoma macdonaldii to produce girdling stem lesions and cause premature ripening on sunflower

\begin{tabular}{|c|c|c|c|c|}
\hline Inoculation method ${ }^{w}$ & $\operatorname{AUDPC}_{\mathrm{DS}}{ }^{\mathrm{x}}$ & Final DS $(\%)^{\mathrm{y}}$ & $\operatorname{AUDPC}_{\mathrm{PR}^{\mathrm{x}}}{ }^{\mathrm{x}}$ & Final PR $(\%)^{y}$ \\
\hline \multicolumn{5}{|l|}{ Auzeville (Auz-07) } \\
\hline Mycelium & $64.15 \mathrm{a}^{\mathrm{z}}$ & $96.3 \mathrm{a}$ & $24.04 \mathrm{a}$ & $64.0 \mathrm{a}$ \\
\hline Buried residues and some left at soil surface & $3.17 \mathrm{~b}$ & $16.7 \mathrm{~b}$ & $0.27 \mathrm{~b}$ & $4.0 \mathrm{c}$ \\
\hline Control & $0 \mathrm{~b}$ & $0 \mathrm{~b}$ & $0 \mathrm{~b}$ & $0 \mathrm{c}$ \\
\hline \multicolumn{5}{|l|}{ En-Crambade (EC-07) } \\
\hline Mycelium & $45.18 \mathrm{a}$ & 96.7 a & $1.49 \mathrm{a}$ & $32.2 \mathrm{~b}$ \\
\hline Buried residues and some left at soil surface & $0.16 \mathrm{~b}$ & $1.7 \mathrm{~b}$ & $0 \mathrm{a}$ & $0 \mathrm{c}$ \\
\hline Control & $0 \mathrm{~b}$ & $0 \mathrm{~b}$ & $0 \mathrm{a}$ & $0 \mathrm{c}$ \\
\hline \multicolumn{5}{|l|}{ Grignon (Gri-08) } \\
\hline Mycelium & $57.00 \mathrm{a}$ & $82.7 \mathrm{a}$ & $2.49 \mathrm{a}$ & $82.6 \mathrm{a}$ \\
\hline Residues left at soil surface & $6.59 \mathrm{~b}$ & $35.1 \mathrm{~b}$ & $0.91 \mathrm{~b}$ & $30.4 \mathrm{~b}$ \\
\hline Buried residues & $0 \mathrm{c}$ & $0 \mathrm{c}$ & $0 \mathrm{c}$ & $0 \mathrm{c}$ \\
\hline Control & $0 \mathrm{c}$ & $0 \mathrm{c}$ & $0 \mathrm{c}$ & $0 \mathrm{c}$ \\
\hline
\end{tabular}

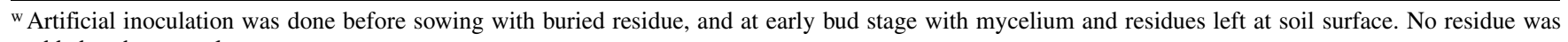
added to the control treatment.

${ }^{x}$ Area under disease progress curve (AUDPC) was calculated according to Campbell and Madden (1990) up to 83 days postinoculation. Disease severity (DS) is based on percentage of plants with girdling stem base lesions, and premature ripening (PR) is based on percentage of plants totally wilted before physiological maturity.

${ }^{y}$ Final DS and final PR were taken 83 days from the start of epidemic.

${ }^{\mathrm{z}}$ Within each column, mean values followed by different letters are significantly different from one another based on $\mathrm{LSD}_{0.05}$. 
with $1 \times 10^{3}$ spores $/ \mathrm{ml}$. AUDPC PR $_{\text {for PR }}$ over time up to 78 DPI varied significantly $(P=0.0005)$ according to spore concentrations and mycelium inoculation. The lowest and highest AUDPC $_{\mathrm{PR}}$ were observed with $1 \times 10^{3}$ and $1 \times 10^{6}$ spores $/ \mathrm{ml}$, respectively (Fig. 3). The final percentage of PR plants also differed significantly $(P<$ $0.0001)$ according to inoculation method. The total number of PR plants increased linearly with increasing levels of inoculum concentration. Final PR was lowest and highest when plants were inoculated with 1 $\times 10^{3}$ and $1 \times 10^{6}$ spores $/ \mathrm{ml}$, respectively. No significant differences were observed between inoculation with mycelium and 1 $\times 10^{6}$ spores $/ \mathrm{ml}$. All PR plants showed girdling stem base lesions, and Pearson's correlation coefficient for the percentage of plants with girdling stem base lesions (Fig. 2) and PR was $r=0.871(P<0.0001)$ at $49 \mathrm{DPI}$ and increased to $r=0.941(P<$ $0.0001)$ at $78 \mathrm{DPI}$

\section{DISCUSSION}

This study focused on producing sunflower premature ripening under greenhouse conditions to obtain a better understanding of how inoculum type and inoculation methods affected disease development. Results showed that infected residues, mycelium, and conidia of $P$. macdonaldii can infect sunflower stem bases and roots, and induce symptoms that may lead to plant death. These findings agree with earlier studies in other sunflower production areas $(7,22,23)$. The existence of a fungal complex (P. macdonaldii, Fusarium sp., and Macrophomina phaseoli) has long been associated with sunflower PR $(7,23)$. This study showed that $P$. macdonaldii alone can cause PR.

Symptoms and development of the disease after inoculation at the stem base, and the time to $\mathrm{PR}$ in the greenhouse were similar to field results with cv. Heliasol RM and other cultivars under a high level of $\mathrm{N}$ fertilization and a limited postanthesis water regime (29). Inoculation with mycelium and spore concentrations of $1 \times 10^{5}$ and $1 \times 10^{6}$ spores $/ \mathrm{ml}$ were identified as the methods that significantly $(P<$ 0.0001 ) promoted establishment of the pathogen leading to $\mathrm{PR}$ while inoculation with spore concentrations at $1 \times 10^{3}, 1 \times$ $10^{4}$ spores $/ \mathrm{ml}$, buried residues, or residues on the soil surface resulted in low percentages of PR. These results are in agreement with a previous report (22), where inoculation with conidia of $P$. macdonaldii at the stem base induced more girdling lesions and PR than inoculation with infected residues. Stem base inoculation methods with mycelium or a spore suspension at 1 $\times 10^{6}$ spores $/ \mathrm{ml}$ could thus be suitable for the efficient evaluation of a large number of sunflower genotypes for resistance to PR. Partial resistance to Phoma black stem has been reported in both field (19) and controlled conditions $(2,25,26)$. Programs screening for resistance to Phoma black stem at present use spore suspensions at

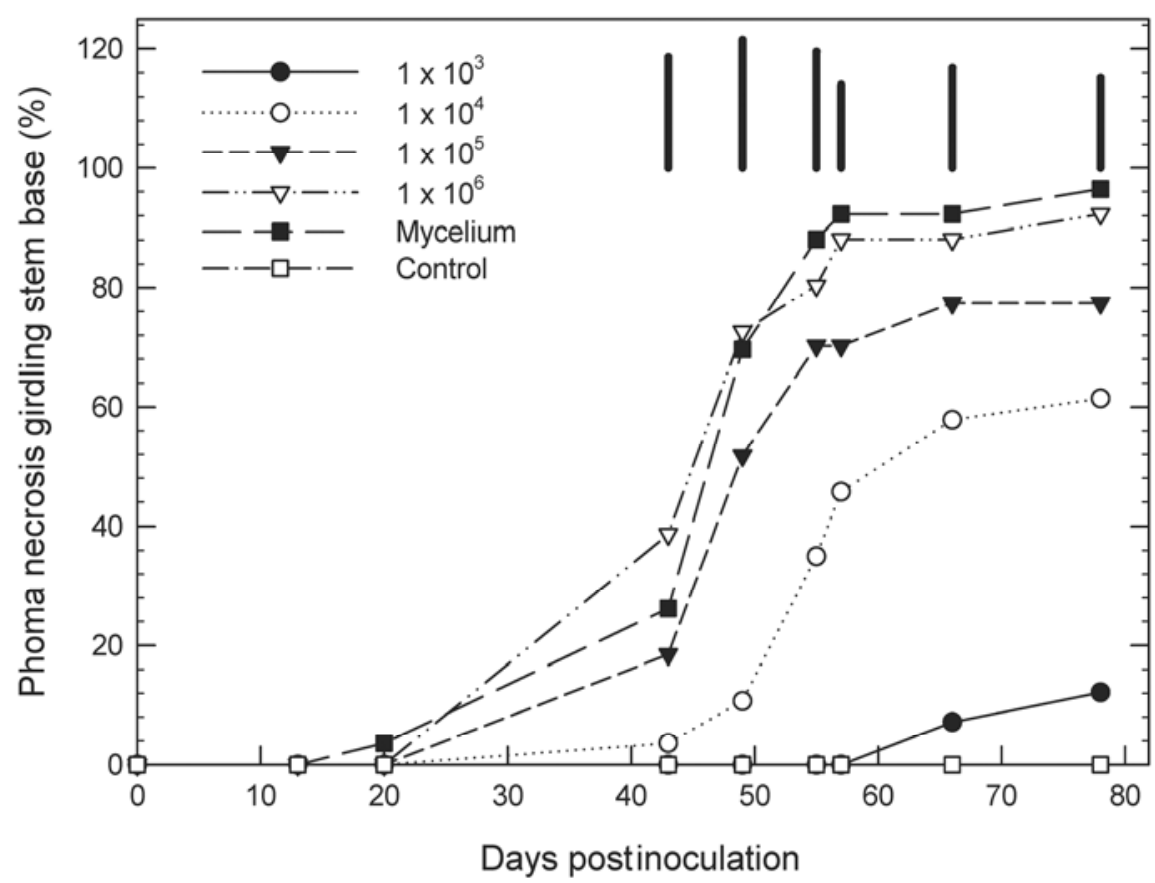

Fig. 2. Effect of artificial inoculation at stem base with mycelium and different conidia concentrations of Phoma macdonaldii on the percentage of sunflower cv. Heliasol RM with girdling stem base lesions in Auzeville in 2008. Vertical bars indicate the $\mathrm{LSD}_{0.05}$ value.

Table 2. Isolations of Phoma macdonaldii from sunflower plants inoculated by different methods

\begin{tabular}{|c|c|c|c|c|c|c|c|c|c|c|}
\hline \multirow[b]{3}{*}{ Inoculation methods $\mathbf{s}^{\mathrm{y}}$} & \multirow{3}{*}{$\begin{array}{l}\text { Plant PR } \\
(83 \text { DPI })^{z}\end{array}$} & \multicolumn{9}{|c|}{ Isolation from plants ${ }^{v}$} \\
\hline & & \multicolumn{3}{|c|}{ Stem basew $^{w}$} & \multicolumn{3}{|c|}{ Stem $^{x}$} & \multicolumn{3}{|c|}{ Root } \\
\hline & & Epiderm & Cortex & Pith & Epiderm & Cortex & Pith & Hair & Rhizoderm & Cortex \\
\hline \multicolumn{11}{|l|}{ Auzeville (Auz-07) } \\
\hline Mycelium & $6 / 8$ & $8 / 8$ & $7 / 8$ & $4 / 8$ & $7 / 8$ & $3 / 8$ & $2 / 8$ & $0 / 8$ & $0 / 8$ & $1 / 8$ \\
\hline Buried residues (total) & $1 / 8$ & $2 / 8$ & $2 / 8$ & $2 / 8$ & $2 / 8$ & $1 / 8$ & $1 / 8$ & $2 / 8$ & $2 / 8$ & $2 / 8$ \\
\hline Plants with stem base necrosis & $1 / 4$ & $2 / 4$ & $2 / 4$ & $2 / 4$ & $2 / 4$ & $1 / 4$ & $1 / 4$ & $1 / 4$ & $1 / 4$ & $1 / 4$ \\
\hline $\begin{array}{l}\text { Plants without stem base } \\
\text { necrosis }\end{array}$ & $0 / 4$ & $0 / 4$ & $0 / 4$ & $0 / 4$ & $0 / 4$ & $0 / 4$ & $0 / 4$ & $1 / 4$ & $1 / 4$ & $1 / 4$ \\
\hline Control & $0 / 8$ & $0 / 8$ & $0 / 8$ & $0 / 8$ & $0 / 8$ & $0 / 8$ & $0 / 8$ & $0 / 8$ & $0 / 8$ & $0 / 8$ \\
\hline \multicolumn{11}{|l|}{ En-Crambade (EC-07) } \\
\hline Mycelium & $17 / 27$ & $25 / 27$ & $2 / 2$ & $16 / 27$ & $\ldots$ & $\ldots$ & $\ldots$ & $8 / 27$ & $\ldots$ & $\ldots$ \\
\hline Buried residues (total) & $0 / 15$ & $5 / 15$ & $\ldots$ & $0 / 15$ & $\ldots$ & $\ldots$ & $\ldots$ & $1 / 15$ & $\ldots$ & $\ldots$ \\
\hline Plants with stem base necrosis & $0 / 2$ & $2 / 2$ & $\ldots$ & $0 / 2$ & $\ldots$ & $\ldots$ & $\ldots$ & $0 / 2$ & $\ldots$ & $\ldots$ \\
\hline $\begin{array}{l}\text { Plants without stem base } \\
\text { necrosis }\end{array}$ & $0 / 13$ & $3 / 13$ & $\ldots$ & $0 / 13$ & $\ldots$ & $\ldots$ & $\ldots$ & $1 / 13$ & $\ldots$ & $\ldots$ \\
\hline Control & $0 / 3$ & $0 / 3$ & $\ldots$ & $0 / 3$ & $\ldots$ & $\ldots$ & $\ldots$ & $0 / 3$ & $\ldots$ & $\ldots$ \\
\hline
\end{tabular}

${ }^{\mathrm{v}}$ Values are number of plants from which P. macdonaldii was recorded out of total number of plants sampled.

${ }^{w}$ At the leading edge of the infection.

${ }^{x}$ At 4 to $5 \mathrm{~cm}$ above stem base necrosis.

y Artificial inoculation was done before sowing with buried residue, and at early bud stage with mycelium and residues left at soil surface. No residue was added to the control treatment.

${ }^{\mathrm{z}} \mathrm{PR}=$ premature ripening. Mortality data at 83 days postinoculation (DPI) were analyzed with a logistic model. Auzeville: $\chi^{2}, P=0.0015$ and En-Crambade: $\chi^{2}, P<0.0001$. 
$1 \times 10^{6}$ spores/ml deposited at the intersection of the leaf petiole and the stem (12). The present results indicate that resistance to Phoma black stem and PR could be screened for using the same spore inoculum and plant growth conditions as used in this study. Genotypes with partial resistance could be used in breeding programs to develop durable resistance to sunflower PR.

Disease severity was used in addition to $\mathrm{AUDPC}_{\mathrm{DS}}$ to evaluate efficient inoculation methods with $P$. macdonaldii leading to PR. The relationship between DS and PR was established in greenhouse and field experiments $(5,7,22)$. In this study, a close correlation between the proportion of plants with girdling lesions at the stem base and the final percentage of PR was observed after inoculation with mycelium and spores. DS could be used as an early and quantifiable indicator to estimate plant susceptibility to PR or score resistant genotypes before development of PR. DS would therefore be useful in large-scale breeding programs where single-date assessments are a considerable advantage. Furthermore, considering that pathogen spread is not affected whatever the phenological plant stage (12), it would be valuable to test whether correlations between Phoma girdling and PR can be obtained with early inoculation. The ability to reproduce disease symptoms and sunflower PR in the greenhouse could allow rapid progress in breeding for disease resistance. Screening is most commonly conducted under field conditions; however, field screening has limitations depending on the natural occurrence of suitable environmental conditions and pathogen inoculum, and can usually be conducted only once in a year. Although only a limited number of plants can be evaluated under greenhouse conditions, experiments could be carried out twice a year for a primary screening of genotypes before field evaluation.

Extensive necrosis of stem tissues occurred several centimeters above the leading edge of the infection after inoculation with spores, mycelium, and infected residues on the soil surface. In contrast, plants inoculated with buried infected residues had few stem base lesions, and only plants growing with fragments of residues remaining on the soil surface in Auz-07 and EC-07 showed girdling lesions. The highest AUDPC $_{\text {DS }}$ and DS in Auz-07 with buried residues may have been favored by controlled air humidity and temperature in the greenhouse. Drier climatic conditions in the hoop tunnel of EC-07 with temperatures up to $40^{\circ} \mathrm{C}$ may have restricted fungal spread into the plant $(27,31)$ and could explain why this experimental site resulted in the lowest final PR whatever inoculation method. Plants inoculated with buried residues had well-developed roots, and some developed swollen necrotic areas where roots contacted infected residues. $P$. macdonaldii was isolated from these swollen necrotic roots, but isolations from roots were rare compared to stem tissues with girdling lesions. Microscopic observations showed that hyphae of $P$. macdonaldii spread primarily in the xylem and up into the stem. A previous report noted infection of the vascular system of sunflower seedling roots (1). Despite limited evidence of $P$. macdonaldii in roots, buried residues can result in root infection (21), but hyphae were not observed in the stem after inoculation with buried residues. Although large quantities of inoculum can be buried with infected crop residues, if they do not lead to girdling stem base lesions, they will not cause sunflower PR. These findings provide a better understanding of the origin of sunflower premature death, which appears primarily due to aerial infections at the stem base rather than through root infections.

Maric et al. (14) described the presence of perithecia and pycnidia of P. macdonaldii on residues aged over several years and suggested that residues can be longterm sources of inoculum $(20,24)$. Large quantities of residues on the soil surface in Gri-08 favored PR more than in Auz-07 and EC-07, where very little residue remained. These observations support the concept that disease development largely depends on the quantity of infected residues remaining on the soil surface (11). In southwestern France, sunflower is mainly planted in rotation with wheat. Short rotations and reduced soil tillage of wheat crops have tended to increase the amount of inoculum by leaving infected sunflower residues on the soil surface. As soil tillage determines the vertical distribution of residues, it must have a pronounced effect on disease development. Contact of plant residue with soils, especially when buried, promotes greater microbial activity, which accelerates decomposition (17). Residues in no-till systems decompose more slowly than residues under reduced-tillage systems. Buried residues brought back to the soil surface were found to be less infectious than nonburied residues (24). Thus, residues in no-till systems will favor the survival of $P$. macdonaldii and inoculum production over longer periods of time.

In conclusion, sunflower PR is primarily due to aerial infections of the stem base and not root infection. Residues left on the soil surface that produce ascospores, conidia, and mycelium are the main source of $P$. macdonaldii inoculum. Cultural practices such as tillage, residue management, and crop rotation that can minimize the quantity and duration of these residues should reduce stem base infection and frequency of sunflower PR. In addition, this study showed that artificial inoculation at the stem base with high conidia concentrations and mycelium of $P$. macdonaldii could be used for screening genotypes for partial resistance to PR. Inoculation with 1 $\times 10^{6}$ spores $/ \mathrm{ml}$ was the most effective

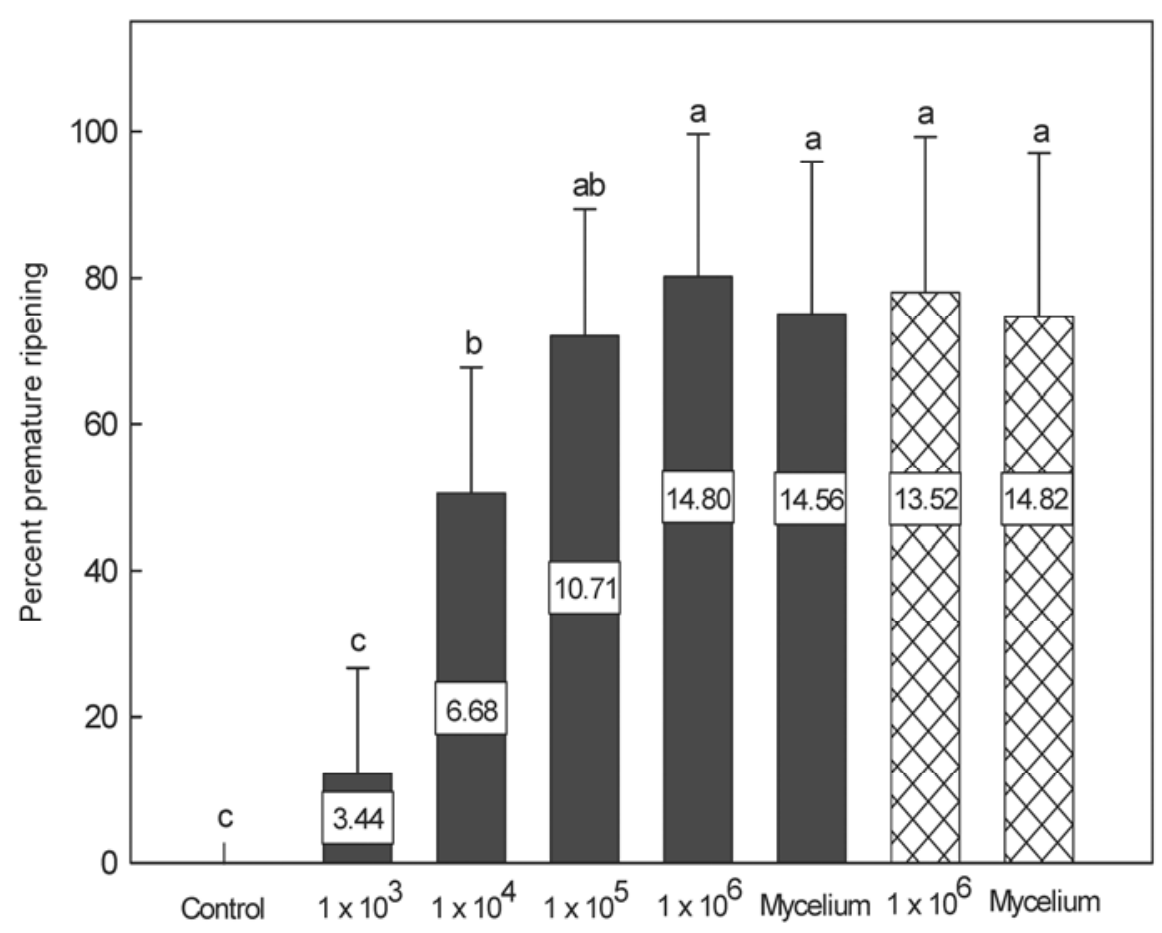

Fig. 3. Effect of artificial inoculation at stem base with different conidia concentrations and mycelium of Phoma macdonaldii on final percentage of plants with premature ripening at 78 days postinoculation (DPI) in Auzeville in 2008 (black bars) and 2009 (squared bars). For each bar, vertical lines represent standard error of the mean. Premature ripening (\%) means with different letters are significantly different by Fisher's protected $\mathrm{LSD}_{0.05}$. Values in bars are area under disease progress curve for premature ripening $\left(\mathrm{AUDPC}_{\mathrm{PR}}\right.$ ) calculated according to Campbell and Madden (4) and based on percentage of plants prematurely ripened from inoculation at 78 DPI. 
method of artificial inoculation and could be applied in screening for resistance to both Phoma black stem and premature ripening.

\section{ACKNOWLEDGMENTS}

This study was supported by CETIOM, MidiPyrénées government, and PROMOSOL association. We are grateful to the technical staff (M. Labarrère, P. Perrin, A. Doumenc, J. Sanyas, A. Bammé, V. Demoisson) for their assistance in experimental procedures and Y. Martinez (Microscopy Platform, Institut Fédératif de Recherche 40) for his technical assistance concerning the confocal microscopy. We also thank N. Seguin, A. Sirvent, A. Pauvert, and C. Jourda for their collaboration. Thanks to F. Vear for careful English checking.

\section{LITERATURE CITED}

1. Abou Al Fadil, T., Jauneau, A., Martinez, Y., Rickauer, M., and Dechamp-Guillaume, G. 2009. Characterisation of sunflower root colonisation by Phoma macdonaldii. Eur. J. Plant Pathol. 124:93-103.

2. Bert, P. F., Dechamp-Guillaume, G., Serre, F., Jouan, I., Tourvieille de Labrouhe, D., Nicolas, P., and Vear, F. 2004. Comparative genetic analysis of quantitative traits in sunflower (Helianthus annuus L.) 3. Characterization of QTL involved in resistance to Sclerotinia sclerotiorum and Phoma macdonaldii. Theor. Appl. Genet. 109:865-874

3. Boerema, G. H. 1970. Additional notes on Phoma herbarum. Persoonia 6:15-48.

4. Campbell, C., and Madden, L. V. 1990. Introduction to Plant Disease Epidemiology. John Wiley \& Sons, New York.

5. Carson, M. 1991. Relationship between Phoma black stem severity and yield losses in hybrid sunflower. Plant Dis. 75:1150-1153.

6. Debaeke, P., and Pérès, A. 2003. Influence of sunflower (Helianthus annuus L.) crop management on Phoma back stem (Phoma macdonaldii Boerema). Crop Prot. 22:741-752.

7. Donald, P. A., Venette, J. R., and Gulya, T. J. 1987. Relationship between Phoma macdonaldii and premature death of sunflower in North Dakota. Plant Dis. 71:466-468.

8. Frezzi, M. J. 1968. Leptosphaeria lindquistii $\mathrm{n}$. sp., forma sexual de Phoma oleracea var. helianthi-tuberosi Sacc., hongo causal de la 'mancha negra del tallo' del girasol (Helianthus annuus L.), in Argentine. Patol. Veg. 5:73-80.

9. Gomez, K. A., and Gomez, A. A. 1984. Statis- tical Procedures for Agricultural Research, 2nd ed. John \& Sons, New York

10. Gulya, T., Rashid, K. Y., and Masirevic, S. M. 1997. Sunflower diseases: Phoma black stem. Pages 319-322 in: Sunflower Technology and Production. A. A. Schneiter, ed. Agronomy Monogr. no. 35, Madison, WI.

11. Jouffret, P. 2005. Sustainable control strategy against Phomopsis and Phoma. (In French.) Oléoscope 80:28-30.

12. Larfeil, C. 2003. La maladie des taches noires du tournesol causée par Phoma macdonaldii Boerema: Variabilité phénotypique et moléculaire - Evaluation de la sensibilité des génotypes à la maladie - Modalités de l'infection. Ph.D. thesis. Toulouse National Polytechnic Institute, Toulouse, France.

13. Maric, A., and Schneider, R. 1979. Black spot of sunflowers in Yugoslavia and its causal agent Phoma macdonaldii Boerema. Phytopathol. Zeitschrift. 94:226-233.

14. Maric, A. D., Masiveric, S., and Fayzalla, S. 1981. Presence of Leptosphaeria lindquistii Frezzi, last stade of Phoma macdonaldii Boerema, causal agent of Black spot of sunflowers in Yugoslavia. (In Serbo-Croatian.) Zastija Bilja. 32:329-344

15. McDonald, W. C. 1964. Phoma black stem of sunflower. Phytopathology 54:492-493.

16. Miric, E., Aitken, E. A. B., and Goulter, K. C. 1999. Identification in Australia of the quarantine pathogen of sunflower Phoma macdonaldii (Teleomorph: Leptosphaeria lindquistii). Aust. J. Agric. Res. 50:325-332.

17. Parr, J. F., and Papendick, R. I. 1978. Factors affecting the decomposition of crop residues by microorganisms. Pages 101-129 in: Crop Residue Management Systems. W. R. Oschwald, ed. American Society of Agronomy, Madison, WI.

18. Penaud, A., and Pérès, A. 1994. The Phoma of sunflower. (In French.) Oléoscope 15, 37 p.

19. Pérès, A., Allard, A. M., Deverchère, J., and Penaud, A. 1994. The Phoma of sunflower: Study of fungicide protection in the field. (In French.) Pages 1179-1185 in: Proc. IV Int. Plant Dis. Conf., Bordeaux, France.

20. Pérès, A., and Lefol, C. 1996. Phoma macdonaldii Boerema: Elements of biology and development of a method of artificial contamination in controlled conditions. (In French.) Pages 687-693 in: Proc. XIV Int. Sunflower Conf., Beijing, China

21. Pérès, A., and Le Sourne, V. 2000. The Phoma of sunflower: A study of pseudothecia and pycnidia in the contamination process. (In French.) Pages 314-321 in: Proc. VI Int. Plant Dis. Conf., Tours, France.

22. Pérès, A., and Poisson, B. 2000. The early withering of sunflower ("dry plant"): The part played by Phoma and study of the contamination. (In French.) Pages 323-329 in: Proc. VI Int. Plant Dis. Conf., Tours, France.

23. Pérès, A., Poisson, B., and Drolon, G. 2000. The dry plant syndrome on sunflower: Study of causes and approach of the incidence. (In French.) Pages 489-495 in: Proc. VI Int. Plant Dis. Conf., Tours, France.

24. Poisson-Bammé, B., and Pérès, A. 2000. Survey of Phoma (Leptosphaeria lindquistii) on sunflower stubbles. (In French.) Pages 331338 in: Proc. VI Int. Plant Dis. Conf., Tours, France.

25. Rachid Al-Chaarani, G., Roustaee, A., Gentz bittel, L., Mokrani, L., Barrault, G., DechampGuillaume, G., and Sarrafi, A. 2002. A QTL analysis of sunflower partial resistance to downy mildew (Plasmopara halstedii) and black stem (Phoma macdonaldii) by the use of recombinant inbred lines (RILs). Theor. Appl. Genet. 104:490-496.

26. Roustaee, A., Barrault, G., DechampGuillaume, G., Lesigne, P., and Sarrafi, A. 2000. Inheritance of partial resistance to black stem (Phoma macdonaldii) in sunflower. Plant Pathol. 49:396-401.

27. Roustaee, A., Costes, S., Dechamp-Guillaume G., and Barrault, G. 2000. Phenotypic variability of Leptosphaeria lindquistii (anamorph: Phoma macdonaldii), a fungal pathogen of sunflower. Plant. Pathol. 49:227-234.

28. Sackston, W. E. 1950. Sunflower diseases in Manitoba in 1949. Can. Plant Dis. Surv. 28:3135.

29. Seassau, C., Dechamp-Guillaume, G., Mestries, E., and Debaeke, P. 2010. Nitrogen and water management can limit premature ripening of sunflower induced by Phoma macdonaldii. Field Crops Res. 115:99-106.

30. Velazquez, P. D., and Formento, N. 2000. Effect of nitrogen fertilization on stem black spot (Phoma oleracea var. helianthi-tuberosi Sacc.) in four genotypes of sunflower (Helianthus annuus L.). AgriScientia 17:41-47.

31. Weeraratne, W., and Priyantha, M. 2003. First report of Phoma black stem of sunflower in Sri Lanka and its management. Ann. Sri Lanka Dep. Agric. 5:263-270. 